\title{
OSMOLALITY AND HYDROGENIONIC POTENTIAL OF MAINTENANCE SOLUTIONS FOR INTRAVENOUS ADMINISTRATION IN INFANTS AND PRESCHOOLERS
}

Daniele Porto Barros; Victória Rodrigues Tavares; Priscilla SC Onofre; Renato Lopes Souza; Laura Naspitz; Mavilde LG Pedreira; Maria Angélica S Peterlini

Department of Pediatric Nursing - Paulista School of Nursing - Department of Pediatric - Paulista School of Medical - Federal University of São Paulo

\section{BACKGROUND}

The intravenous therapy includes the infusion of drugs and solutions into the venous system through devices called catheters. Despite all advantages of therapy, some characteristics of the infused solutions, such as extreme values of hydrogenionic potencial $(\mathrm{pH})$ and osmolality, increase the risk of development of local complications such as phlebitis, infiltration and extravasation.

\section{AlM}

To verify the $\mathrm{pH}$ and osmolality of solutions composed by glucose and electrolytes commonly used for peripheral intravenous administration in infants and preschoolers.

\section{METHOD}

- Place of study: LEEnf-UNIFESP.

- Collection period: May to June 2017.

- Conditions: luminosity (Ix $99.32 \pm 24.17$ ), temperature (22.88 \pm $\left.0.58{ }^{\circ} \mathrm{C}\right)$ and relative humidity of air $(60.99 \pm 5.44 \%)$ were controlled.

- Compositions of solutions: Glucose 5\%, Sodium chloride $20 \%$, Potassium chloride 19,1\%, Magnesium sulfate $10 \%$ and Calcium gluconate $10 \%$.

$\checkmark$ The solutions were prepared in a Biogreen $\AA$ laminar flow hood, using aseptic technique.

- Were verified pH and osmolality of solutions in their commercial presentations and of eight different types of solutions used in infants and preschoolers in a preoperative fasting condition and in the treatment of septic shock.

Picture 1 - Solutions composed by glucose and electrolytes that participated in the composition of solutions prescribed for children by age group, in milliliters.

\begin{tabular}{|c|c|c|c|c|c|}
\hline $\begin{array}{l}\text { Types of solutions } \\
\text { prescribed by age group }\end{array}$ & 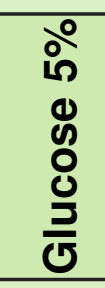 & 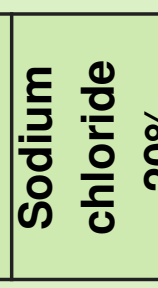 & 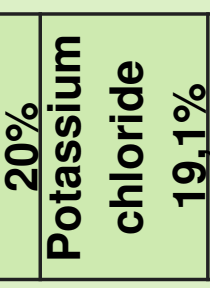 & 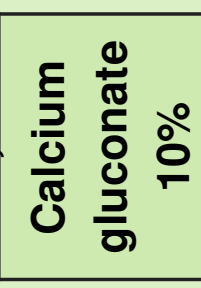 & 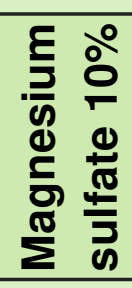 \\
\hline \multicolumn{6}{|l|}{ Infants } \\
\hline Solution - preoperative 1 & 520 & 4.8 & 5.2 & & \\
\hline Solution - preoperative 2 & 500 & 20 & 5 & & \\
\hline Solution - septic shock 1 & 520 & 4.8 & 5.2 & 5.5 & 2 \\
\hline Solution - septic shock 2 & 500 & 20 & 5 & 5.5 & 2 \\
\hline \multicolumn{6}{|l|}{ Preschoolers } \\
\hline Solution - preoperative 2 & 500 & 4.5 & 5.0 & & \\
\hline Solution - preoperative 2 & 500 & 20 & 5 & & \\
\hline Solution - septic shock 1 & 500 & 4.5 & 5 & 7 & 1.8 \\
\hline Solution - septic shock 2 & 500 & 20 & 5 & 7 & 1.8 \\
\hline
\end{tabular}

\section{RESULTS}

The solutions were prepared and analyzed in triplicate, resulting in 168 verifications: 87 of $\mathrm{pH}$ and 81 of osmolality.

Table I - Average, minimum, maximum and standard deviation values of osmolality and hydrogenionic potential of solutions in their commercial presentations

\begin{tabular}{|c|c|c|c|c|c|c|}
\hline \multirow[b]{2}{*}{ Solutions } & \multicolumn{3}{|c|}{ Osmolality (mOsm/kg) } & \multicolumn{3}{|c|}{ Hydrogenionic potential } \\
\hline & Average ( $\pm \mathrm{SD}$ ) & $\min$. & $\max$. & $\begin{array}{c}\text { Average } \\
( \pm S D)\end{array}$ & $\min$. & $\max$. \\
\hline Glucose $5 \%$ & $288.00( \pm 3.46)$ & 286 & 292 & $4.61( \pm 0.19)$ & 4.40 & 4.76 \\
\hline Sodium chloride $20 \%$ & - & - & - & $5.98( \pm 0.43)$ & 5.49 & 6.23 \\
\hline Potassium chloride $19,1 \%$ & - & - & - & $5.82( \pm 0.20)$ & 5.65 & 6.04 \\
\hline Magnesium sulfate $10 \%$ & $474.33( \pm 32.62)$ & 446 & 510 & $5.90( \pm 0.59)$ & 5.48 & 6.57 \\
\hline Calcium gluconate $10 \%$ & $314.33( \pm 22.28)$ & 300 & 340 & $6.33( \pm 0.09)$ & 6.23 & 6.41 \\
\hline
\end{tabular}

Osmolality values of solutions for infants and preschoolers ranged from 383 to $667 \mathrm{mOsm} / \mathrm{kg}$ and $\mathrm{pH}$ from 3.99 to 5.83 .

Table 2 - Average, minimum, maximum and standard deviation values of osmolality and hydrogenionic potential of solutions prescribed for peripheral intravenous administration in infants.

\begin{tabular}{|c|c|c|c|c|c|c|}
\hline \multirow[b]{2}{*}{ Solutions } & \multicolumn{3}{|c|}{ Osmolality (mOsm/kg) } & \multicolumn{3}{|c|}{ Hydrogenionic potential } \\
\hline & Average $( \pm S D)$ & $\min$. & $\max$ & $\begin{array}{c}\text { Average } \\
( \pm S D)\end{array}$ & $\min$. & $\max$. \\
\hline $\begin{array}{l}\text { Solution } \\
\text { preoperative } \\
\text { condition } 1\end{array}$ & $432.56( \pm 45.9)$ & 405.00 & 514.00 & $4.29( \pm 0.35)$ & 4.09 & 5.21 \\
\hline $\begin{array}{l}\text { Solution } \\
\text { preoperative } \\
\text { condition } 2\end{array}$ & $632.78( \pm 21.43)$ & 608.00 & 643.00 & $4.11( \pm 0.05)$ & 4.04 & 4.19 \\
\hline $\begin{array}{l}\text { Solution for treatment } \\
\text { of septic shock } 1\end{array}$ & $415.67( \pm 5.41)$ & 410.00 & 427.00 & $5.42( \pm 0.26)$ & 5.06 & 5.83 \\
\hline $\begin{array}{l}\text { Solution for treatment } \\
\text { of septic shock } 2\end{array}$ & $599.22( \pm 21.05)$ & 571.00 & 619.00 & $5.12( \pm 0.07)$ & 5.04 & 5.28 \\
\hline
\end{tabular}

Table 3 - Average, minimum, maximum and standard deviation values of osmolality and hydrogenionic potential of solutions prescribed for peripheral intravenous administration in preschoolers.

\begin{tabular}{|c|c|c|c|c|c|c|}
\hline \multirow[b]{2}{*}{ Solutions } & \multicolumn{3}{|c|}{ Osmolality (mOsm/kg) } & \multicolumn{3}{|c|}{ Hydrogenionic potential } \\
\hline & Average $( \pm S D)$ & $\min$. & $\max$. & $\begin{array}{l}\text { Average } \\
( \pm S D)\end{array}$ & $\min$. & $\max$ \\
\hline $\begin{array}{l}\text { Solution } \\
\text { preoperative } \\
\text { condition } 1\end{array}$ & $405.00( \pm 3.35)$ & 400.00 & 410.00 & $4.11( \pm 0.13)$ & 3.99 & 4.35 \\
\hline $\begin{array}{l}\text { Solution } \\
\text { preoperative } \\
\text { condition } 2\end{array}$ & $610.22( \pm 14.68)$ & 596.00 & 635.00 & $4.11( \pm 0.07)$ & 4.01 & 4.17 \\
\hline $\begin{array}{l}\text { Solution for treatment } \\
\text { of septic shock } 1\end{array}$ & $409.56( \pm 19.73)$ & 383.00 & 435.00 & $5.07( \pm 0.11)$ & 4.90 & 5.24 \\
\hline $\begin{array}{l}\text { Solution for treatment } \\
\text { of septic shock } 2\end{array}$ & $605.00( \pm 12.31)$ & 576.00 & 616.00 & $5.12( \pm 0.05)$ & 5.08 & 5.20 \\
\hline
\end{tabular}

\section{CONCLUSION}

- Osmolality values were at the upper tolerable limit for peripheral intravenous administration.

- $\quad$ pH values were at the lower tolerable limit.

Higher osmolality: in the presence of Sodium chloride $20 \%$ and Potassium chloride $19,1 \%$.

Lower osmolality: dilution in Glucose 5\%.

More acid pH: in the presence of a lower concentration of electrolytes. 